\title{
ESTUDO RETROSPECTIVO DE 98 FELINOS SUBMETIDOS À ACUPUNTURA ATENDIDOS EM SERVIÇO DE REABILITAÇÃO E DOR CRÔNICA
}

\section{RETROSPECTIVE STUDY OF 98 CATS SUBMITTED TO ACUPUNCTURE ATTENDED AT A REHABILITATION AND CHRONIC PAIN CONTROL SERVICE}

\author{
Nuno Emanuel Oliveira Figueiredo ${ }^{1 *}$ \\ Jean Guilherme Fernandes Joaquim² \\ Stelio Pacca Loureiro Luna ${ }^{1}$ \\ Maria Luísa Buffo de Cápua² \\ Bianca Paiva Rodrigues dos Santos ${ }^{1}$ \\ ${ }^{1}$ Faculdade de Medicina Veterinária e Zootecnia - UNESP, Botucatu, SP, Brasil. \\ 2Instituto Bioethicus, Botucatu, SSP, Brasil. \\ *Autor para correspondência - emmanunno@gmail.com
}

\section{Resumo}

Este estudo retrospectivo avaliou os casos clínicos de felinos atendidos em Serviço de Reabilitação e Dor Crônica, durante 13 anos, totalizando 98 prontuários. Os objetivos deste estudo foram caracterizar o perfil do paciente atendido e avaliar a eficácia da acupuntura (AP) e dos métodos associados a esta técnica no tratamento de doenças em felinos e respectiva evolução clínica. Constatou-se que 69,7\% dos pacientes melhoraram após tratamento com AP, 16,7\% não apresentaram resposta significativa e 13,6\% vieram a óbito. Não foram registrados casos de piora relacionados ao tratamento com AP, embora, dos nove animais que vieram a óbito, três foram devido à progressão da doença primária tratada exclusivamente com AP. As principais afecções tratadas foram relacionadas ao sistema nervoso - sobretudo trauma medular e encefálico - em que se registrou uma melhora em 53,7\% dos animais. O tratamento foi considerado eficaz quando os pacientes recuperaram a capacidade normal de deambulação e em casos de doenças medulares quando o escore neurológico mudou de IV (grave) ou V (muito grave) para I (normal) ou II (leve alteração). A técnica de agulha seca foi a mais utilizada para os tratamentos, com 94,7\% de prevalência, seguida da eletroacupuntura $(30,6 \%)$ e da laserpuntura $(21,4 \%)$. O número de sessões totais de AP foi em média de $6,97 \pm 14,97$, sendo as mesmas semanais. Conclui-se que o estímulo de acupontos utilizando diferentes métodos foi eficiente para tratar a maioria dos gatos com diversas condições clínicas, evidenciando que esta espécie provavelmente responde tão bem como os cães após o tratamento de AP.

Palavras-chave: medicina tradicional chinesa; gatos; pesquisa primária; clínica; neurologia.

\section{Abstract}


This is a retrospective survey with the aim of investigate the clinical cases of cats attended at a rehabilitation and chronic pain control service during 13 years. The aim of this study was to analyze the efficacy and methods of acupuncture, evaluate clinical evolution and the patient profile in 98 clinical charts. The clinical outcome data showed that $69.7 \%$ of patients improved after treatment with acupuncture, $16.7 \%$ had no significant response and $13.6 \%$ died. No patient had worsening, despite three of nine animals that died were due to progression of the primary disease treated only with acupuncture. The main indications for the use of acupuncture in cats at the clinic were neurological diseases - particularly trauma in the spinal cord and brain - where there was an improvement in $53.7 \%$ of the animals. Treatment was considered successful in neurological cases, when animals regained independent locomotion and in cases of spinal disease, when neurological scores changed for IV or V to I or II. Dry needle acupuncture was the most used technique for the treatment, with $94.7 \%$ of prevalence, followed by electroacupuncture $(30.6 \%)$ and laserpuncture $(21.4 \%)$. The mean number of acupuncture sessions were $6.97 \pm 14.97$. It was concluded that acupuncture was efficient to treat most of the cats with different clinical conditions, showing that these species probably respond as well as dogs after acupuncture treatment.

Keywords: chinese traditional medicine; cats; primary research; clinics; neurology.

Recebido em: 18 de fevereiro de 2017

Aceito em: 13 de novembro de 2017

\section{Introdução}

As evidências da eficiência de uma terapia são obtidas com revisões desenvolvidas a partir de estudos primários, que são do tipo descritivo, analítico, retrospectivo e prospectivo. Os estudos retrospectivos são análises clínicas primárias, que consistem no levantamento de prontuários e dados clínicos e são baseados na evolução do paciente, sem um protocolo previamente padronizado de tratamento ${ }^{(1)}$.

A acupuntura (AP) é uma terapia oriunda da Medicina Tradicional Oriental (MTO) que estimula pontos específicos do corpo, denominados acupontos. Apresenta diversas aplicações clínicas devido ao amplo mecanismo de ação que envolve componentes neurológicos, endócrinos e humorais ${ }^{(2)}$. Os acupontos são escolhidos de acordo com os padrões de enfermidades estudadas pela MTO e/ou indicações relacionadas ao seu efeito comprovado através de estudos científicos.

Diversas pesquisas em medicina veterinária baseada em evidências comprovaram os efeitos terapêuticos da AP em várias enfermidades. No sistema gastrointestinal a eletroacupuntura (EA) aumentou o peristaltismo gastrointestinal em cães $^{(3)}$, sendo que em outro estudo a AP e EA aumentaram a pressão intragástrica de cães $^{(4)}$. No sistema cardiovascular a AP foi eficaz na ressuscitação cardiorrespiratória, com efeitos simpaticomiméticos em cães ${ }^{(5)}$, além de produzir efeitos antiarrítmicos nessa espécie ${ }^{(6)}$. A AP também foi bem-sucedida no tratamento de trombocitopenia imunomediada em cães, quando associada à fitoterapia chinesa ${ }^{(7)}$, através de um mecanismo de ação humoral.

O presente estudo mostra o levantamento retrospectivo dos casos clínicos de felinos atendidos em Serviço de Reabilitação e Dor Crônica, no período de 2000 a 2013, totalizando 98 casos. Os objetivos deste estudo foram: 1 . caracterizar o perfil do paciente atendido; 2. avaliar a eficácia da AP e dos 
métodos associados a esta técnica no tratamento de doenças em felinos e respectiva evolução clínica.

\section{Materiais e Métodos}

O estudo foi aprovado pela Comissão de Ética no Uso de Animais (CEUA) da instituição, sob o protocolo $n^{\circ} 150 / 2014$. Todos os proprietários autorizaram a realização do estudo em seus animais por meio de um termo de consentimento.

Os 98 prontuários dos animais atendidos no Serviço de Reabilitação e Dor Crônica foram organizados em ordem cronológica e avaliados quanto a sua completude. Para cada categoria de informação foi realizada análise descritiva com percentual de ocorrência. Os protocolos com preenchimento completo $(67,3 \%)$ foram categorizados segundo a espécie, raça, idade, sexo, doença, tratamentos anteriores e resposta aos mesmos, tratamentos concomitantes, duração do tratamento com AP, número total de sessões de AP, tipo de tratamento (técnicas e as suas associações) e evolução clínica.

Os protocolos com dados incompletos $(32,7 \%)$, tratamentos descontinuados ou outros problemas que invalidassem o estudo dos dados de forma fidedigna foram apenas utilizados apenas para as categorias idade, raça, doença e terapias utilizadas. A maioria dos pacientes foram avaliados e, algumas vezes, previamente tratados pelas equipes dos Serviços de Cirurgia e Clínica de Pequenos Animais antes de serem encaminhados ao Serviço de Reabilitação e Dor Crônica. Outros casos foram encaminhados diretamente para este serviço. A maioria dos tratamentos foi realizada apenas uma vez por semana, durante aproximadamente 20 minutos.

Os critérios de inclusão dos animais em estudo foram baseados no exame físico e exames complementares, como diagnóstico por imagem- radiografias simples ou contrastadas, ressonância magnética (RM) ou tomografia computadorizada, evolução dos parâmetros hematológicos, séricos e urinários e, em casos específicos, caracterização histopatológica de áreas alteradas, identificação de microrganismos envolvidos na afecção e análise de líquido cefalorraquidiano. Não foi necessária a presença de um ou todos os exames complementares para inclusão dos casos na presente revisão.

Em casos de doenças neurológicas, a cada sessão semanal era realizado um novo exame neurológico. O tratamento foi considerado eficaz quando os pacientes foram capazes de recuperar seu controle neuromuscular ou a capacidade normal de deambulação - conforme proposto por Joaquim et al. ${ }^{(8)}$, e em casos de doenças medulares quando o escore neurológico mudou de IV (grave) ou V (muito grave) para I (normal) ou II (leve alteração), sendo o III considerada uma alteração relevante (Tabela 1). 
Tabela 1- Graduação das lesões medulares utilizadas para auxiliar na determinação de tratamento adequado para pacientes com enfermidades neurológicas. Adaptado de Joaquim et al . $^{(8)}$

\begin{tabular}{|c|c|}
\hline Grau & Sinais Clínicos \\
\hline I & Cervicalgia ou lombalgia leve, moderada ou severa, sem déficits neurológicos. \\
\hline II & Discreta incoordenação, capacidade de sustentar o próprio peso mantida, episódios \\
\hline & recorrentes de dor, déficit de propriocepção, reflexos espinhais normais ou aumentados. \\
\hline III & $\begin{array}{c}\text { Severa incoordenação, perda da capacidade de sustentar o próprio peso, déficit de } \\
\text { propriocepção, reflexos espinhais normais ou aumentados. }\end{array}$ \\
\hline IV & $\begin{array}{l}\text { Perda da função motora, ausência de propriocepção, reflexos espinhais normais ou } \\
\text { aumentados, resposta a dor profunda mantida. }\end{array}$ \\
\hline $\mathbf{V}$ & Todos os anteriores mais perda do controle da micção e da dor profunda. \\
\hline
\end{tabular}

\section{Resultados e Discussão}

Em estudo de Ueda et al. ${ }^{(9)}$, a espécie felina representou 5,7\% (48) dos animais, enquanto a espécie canina representou 93,8\% das espécies atendidas. Este dado não reflete a atual tendência de crescimento de gatos como animais de companhia no Brasil, que possui uma população de aproximadamente 20 milhões destes felinos, de acordo com o levantamento realizado pela $\mathrm{ABINPET}^{(10)}$. A população de gatos cresce no país, em média, $8 \%$ ao ano, enquanto o número de cães cresce $4 \%$ ao ano.

No período de 2000 a 2013 foram atendidos 98 felinos, 54 machos e 44 fêmeas. A maioria $(87,8 \%)$ era sem raça definida (86 felinos), 5,1\% da raça Siamês, 5,1\% Persa, 1\% Angora e 1\% Americano de pelo curto. A idade média foi $3,8 \pm 4.6$ anos e a faixa etária predominante (34\%) foi a de adultos jovens (entre 1 e 5 anos de idade) (Figura 1).

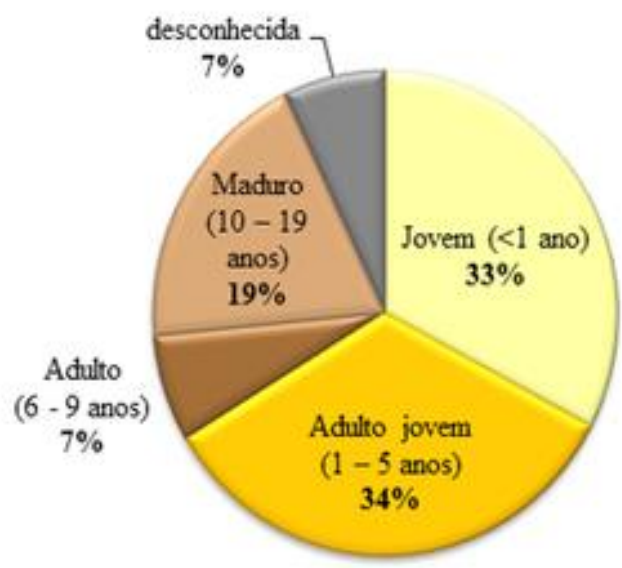

Figura 1 - Categorias etárias dos felinos atendidos no serviço de reabilitação e dor crônica entre 2000 e 2013. Os dados estão expressos em percentual dentro do universo de 98 animais. 
As principais indicações para o tratamento de AP em gatos foram sobretudo casos neurológicos $(68,4 \%)$, e em menor escala casos urinários $(7,1 \%)$, musculoesqueléticos $(5,1 \%)$ e gastrointestinais $(5,1 \%)$ (Figura 2).

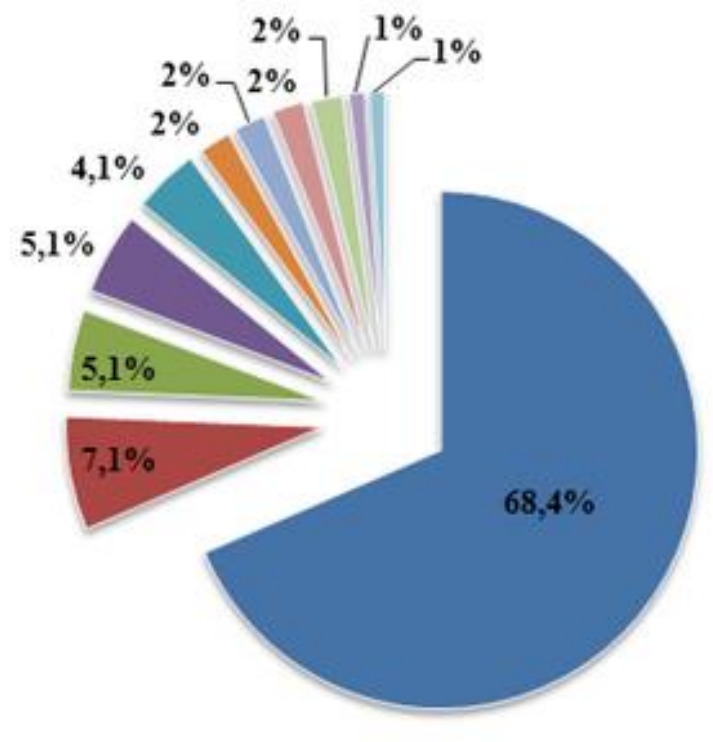

" neurológicas
" urinárias
" musculoesqueléticas
" gastrointestinais
" neoplasias
" dermatológicas
" respiratórias
" endócrinas
" hematológicas
" hepáticas
" comportamentais

Figura 2 - Principais categorias de doenças que comprometem felinos atendidos no serviço de reabilitação e dor crônica entre 2000 e 2013. Os dados estão expressos em percentual dentro do universo de 98 animais.

Uma grande porcentagem de pacientes continuou os tratamentos de AP $(67,3 \%)$, sendo que a maioria dos animais tratados já havia apresentado melhora quando da desistência da continuidade dos tratamentos por parte dos proprietários. Uma possível explicação é o fato dos proprietários estarem felizes com a evolução clínica do animal ou que este não apresentava dor, de forma que a satisfação resulta em abandono do tratamento antes da formalização da alta médica.

Os dados seguintes foram analisados dentro do universo de $66(67,3 \%)$ animais que aderiram ao tratamento com AP e cujos prontuários tinham preenchimento completo, não sendo contabilizados aqueles $(32,7 \%)$ que não continuaram o tratamento após a primeira consulta. Quanto à evolução clínica, 69,7\% dos pacientes tiveram melhora de sinais clínicos após tratamento com AP, 16,7\% não apresentaram resposta significativa e 13,6\% vieram a óbito (Figura 3-A). Dos que morreram, 67,7\% foram por causas não relacionadas à enfermidade tratada e 33,3\% pioraram da doença que motivou o tratamento. Não houve nenhum caso que registrou piora, apesar de três dos nove animais que vieram a óbito serem devido à causa que motivou o tratamento. Para efeitos desse estudo, considerou-se piora uma evolução não natural da enfermidade ou aparecimento de sintoma distinto da queixa inicial. No final do estudo, 3,1\% dos animais ainda estavam sob tratamento no Serviço de Reabilitação e Dor Crônica.

As enfermidades neurológicas prevaleceram, ocorrendo em 68,4\% dos animais; destes, 66,6\% obtiveram melhora dos sinais clínicos, $22,2 \%$ não apresentaram resposta e 11,1\% dos felinos vieram a óbito (Figura 3-B). Esta taxa de recuperação demonstrou que a AP pode ser utilizada no tratamento de doenças neurológicas. Isto ocorreu devido à correta indicação da técnica, que normalmente apresenta boa resposta para afecções neurológicas em geral ${ }^{(11-13)}$. 

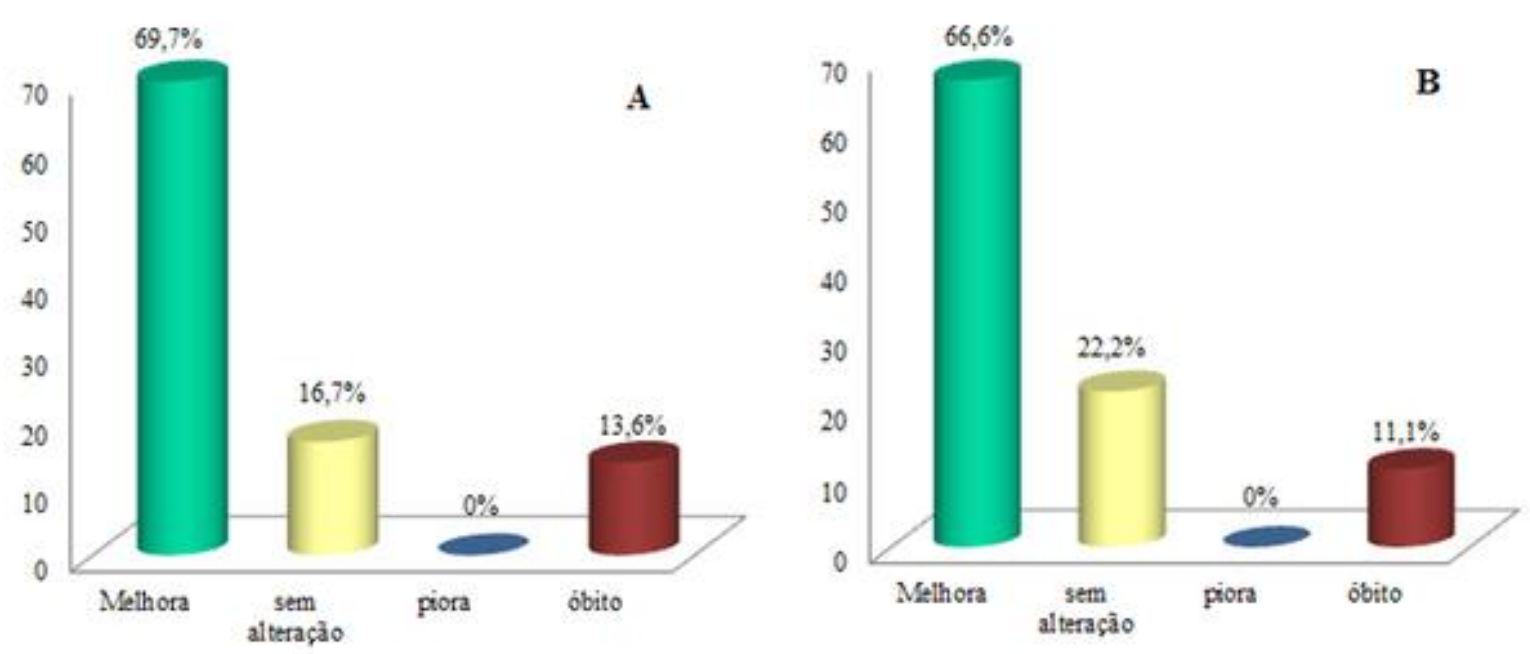

Figura 3 - Evolução clinica dos 66 felinos (A) e dos 45 felinos com doenças neurológicas (B) atendidos no serviço de reabilitação e dor crônica entre 2000 e 2013.

A AP é reconhecida como técnica de tratamento de afecções neurológicas e musculoesqueléticas em pequenos animais, sobretudo em casos associados à dor crônica e neuropática ${ }^{(12,14)}$. Em felinos, a eficácia clínica da AP é relatada em casos de paralisia de nervos periféricos e lesões medulares ${ }^{(15)}$, doença do disco intervertebral (DDIV) ${ }^{(16)}$ e doenças musculoesqueléticas ${ }^{(15,17,18)}$.

A maioria das doenças neurológicas - como sequelas de cinomose, discopatias e traumas medulares são enfermidades de difícil resolução com tratamento médico clínico. Vários graus de paresia ou paralisia decorrem devido às enfermidades supracitadas, podendo ocorrer ainda neuropatias periféricas, mielite e incontinência urinária/fecal neurogênica ${ }^{(19)}$. Neste estudo, dentre as enfermidades neurológicas, a maior prevalência foi de traumatismo (55,2\%): medulares (34 casos) e cranioencefálicos (3). Dos 37 animais que sofreram trauma, $62,2 \%$ aderiram ao tratamento e destes $70 \%$ melhoraram, $17 \%$ não tiveram alteração e $13 \%$ vieram a óbito por outra causa.

Dos 52 animais com problemas neurológicos, $28,8 \%$ foram tratados apenas com AP, em 40,4\% foi associada a EA; em $21,1 \%$ o LASER; em 3,8\% o magneto; em 1,9\% a ozonioterapia; e em 1,9\% a homeopatia. Em alguns casos estas técnicas foram associadas entre si e o número médio de sessões de AP foi de 4,7 por animal. Em alguns pacientes, ao tratamento principal foram associados analgésicos $(4,1 \%$ dos casos, com anti-inflamatórios não esteroidais e esteroidais, opioides e gabapentina) e fármacos complementares para afecções concomitantes $(4,1 \%)$ nenhum outro tipo de fármaco foi utilizado, sendo que os corticoides, quando utilizados, foram em animais que já estavam sob medicação, em doses apenas anti-inflamatórias e cuja terapia foi suprimida nas duas semanas iniciais de tratamento pelo Serviço de Reabilitação e Dor Crônica.

A prevalência do uso da EA foi maior do que a AP manual e a escolha entre cada uma das técnicas foi baseada em achados clínicos como atrofia muscular, lesão neurológica, períodos de paresia/ataxia e ausência ou presença de sensibilidade nociceptiva. O estímulo elétrico pode ser utilizado em casos neurológicos para redução da atrofia muscular, alívio de contraturas e espasmos (pontos-gatilho), bem como da dor ${ }^{(20,21)}$.

Os sinais clínicos mais frequentemente observados nos animais deste estudo foram ataxia, fraqueza, 
déficits de reação postural e hiperestesia nos membros pélvicos. Os padrões neurológicos mais frequentes associados a esses sinais são síndromes lombossacrais, toracolombares e cervicotorácicas $^{(22)}$. Os principais padrões chineses para tais condições são geralmente deficiência de Yang, vazio de Sangue e Qi (energia vital) no Du Mai (Vaso Governador) e síndrome Wei (síndrome de flacidez), que estão associados a sinais como atrofia muscular, paralisia, parestesia, fraqueza, letargia, diarreia, incontinências fecal e urinária, tontura, palpitação e outros sinais ${ }^{(8)}$.

Muitas das condições neurológicas $(35,8 \%)$ observadas no presente trabalho estavam associadas a alterações da micção, nomeadamente incontinência, retenção ou cistite recorrente, sinais que exigem tratamento adequado e cuja resolução é muitas vezes difícil. Na história clínica é importante saber interpretar o que o proprietário relata para saber diferenciar retenção de incontinência urinária.

Os cães com lesão localizada na região L4-S3 podem apresentar sinais clínicos de incontinência. Já os felinos com lesão de neurônio motor inferior (NMI) para membros pélvicos localizados nesta região podem apresentar retenção urinária. Houve 11 felinos atendidos em que esta situação de lesão de NMI com retenção urinária se verificou, com luxações, subluxações ou fraturas respectivamente em: L4, L5-L6, L5-L6-L7, L6-L7, L7-S1 (dois casos), S1, S2-S3, no sacro (três), com amputação de cauda (até $\mathrm{S} 1$ em um caso). Em felinos, as fibras dos nervos pélvicos e hipogástricos atingem o córtex cerebral, enquanto que nos caninos apenas as dos nervos hipogástricos o fazem. Uma lesão lombossacra na medula pode anular o reflexo de micção, apesar do animal perceber a distensão da bexiga como sensação dolorosa, mediada pelas fibras do nervo hipogástrico ${ }^{(23)}$.

A ausência de sensibilidade nociceptiva indica um prognóstico reservado para a recuperação do paciente ${ }^{(11)}$. Cinco felinos com ausência de nocicepção recuperaram a habilidade de andar, com uma média de 6,2 sessões para a melhora da deambulação. A capacidade de recuperação pode estar relacionada à ativação da região Central Geradora de Passos na medula espinhal para a locomoção em mamíferos que, como demonstrado em experimentos com lesão espinhal aguda em felino, é uma locomoção rítmica e não uma sucessão de $\operatorname{reflexos}^{(24)}$ e que pode ser ativada com estímulo adequado.

Em cães com DDIV toracolombar, a associação de EA e tratamento clínico convencional foi mais eficaz que apenas com o uso do tratamento convencional para acelerar a recuperação da deambulação e da percepção nociceptiva. Apesar de relatos clínicos apontarem que a ausência de percepção nociceptiva agrava o prognóstico do animal, 79\% dos cães portadores de discopatia toracolombar tratados com EA foram reabilitados quanto à locomoção ${ }^{(8)}$. A EA foi inclusive mais eficaz que a cirurgia descompressiva tardia na recuperação da locomoção e na melhora dos déficits neurológicos de cães com discopatia ${ }^{(13)}$.

Os proprietários de 15 felinos expressaram como uma das queixas principais a dor, número subestimado, tendo em conta que houve 37 casos de traumas. Animais predadores como os felinos "escondem" a sua dor quando se sentem ameaçados para não evidenciarem a sua vulnerabilidade ${ }^{(25)}$. O manejo da dor por trauma é similar ao da dor pós-cirúrgica. Em felinos, a dor crônica é mais difícil de tratar, também por ser difícil de identificar. A AP tem sido empregada com sucesso para tratar problemas agudos e crônicos de dor com o mínimo de efeitos colaterais sistêmicos ${ }^{(26)}$. A maioria dos felinos tolera bem a colocação da agulha e a AP deve ser considerada uma opção viável para a terapia analgésica, especialmente para condições crônicas em que há poucas opções farmacológicas na espécie felina ${ }^{(27)}$. 
Dos cinco animais com doenças musculoesqueléticas, $80 \%$ melhoraram e $20 \%$ vieram a óbito. Todos foram tratados com AP e dois com LASER. Houve três casos de problemas neurológicos associados a ortopédicos e o único felino que aderiu ao tratamento melhorou. As alterações osteomusculares em cães como as doenças articulares - incluindo displasia coxofemoral (DCF) e osteoartrite (OA) - são tratadas com diferentes técnicas de AP para aliviar a dor ${ }^{(14)}$. Aparentemente, os efeitos benéficos da AP no tratamento de disfunções musculoesqueléticas estão associados com a analgesia secundária à libertação de endorfinas, vasodilatação local, efeitos anti-inflamatórios, alívio da dor em pontosgatilho e alívio da rigidez muscular ${ }^{(17)}$.

As doenças do trato urinário foram observadas em 7,1\% dos animais, segunda categoria de doença mais comum. Três felinos tinham doença renal crônica (DRC) e os outros apresentaram incontinência urinária, cistite e cálculos de estruvita. Todos os felinos que aderiram ao tratamento melhoraram. A MTO pode ajudar a promover uma boa qualidade de vida no felino idoso. No manejo da DRC felina pode-se usar AP, fitoterapia e dietoterapia ${ }^{(28)}$. Giovaninni \& Piai $^{(29)}$ sugerem que a AP como complemento ao tratamento da doença do trato urinário inferior dos felinos (DTUIF) idiopática pode aliviar o sofrimento do doente e minimizar a possibilidade de recidivas da sintomatologia e também pode vir a corroborar na elucidação da hipótese da inflamação neurogênica da bexiga como etiologia da DTUIF idiopática.

Dos cinco felinos com doenças gastrointestinais, todos melhoraram. Uma gata com agenesia anal e hipomotilidade intestinal foi submetida à EA nos acupontos Estômago (E) 21 e E25 e recomeçou a defecar logo após a $1^{\mathrm{a}}$ sessão de AP e após a $3^{\mathrm{a}}$ sessão teve alta. A capacidade de AP para aumentar a motilidade gastrointestinal em humanos e animais tem sido bem documentada. Luna e Joaquim ${ }^{(3)}$ comprovaram que a EA nesses pontos aumenta o peristaltismo gastrointestinal em cães.

Em felinos submetidos à distensão gástrica, a EA no ponto E36 inibiu a frequência do relaxamento transitório do esfíncter inferior do esôfago ${ }^{(30)}$ e a EA em Pericárdio (PC) 6 reduziu o refluxo gastroesofágico $^{(31)}$. Em gatos com esofagite induzida por miotomia, a EA de alta frequência em E36 aumentou a pressão do esfíncter inferior do esôfago, bem como a motilidade do esôfago ${ }^{(32)}$.

Dos dois pacientes oncológicos, um veio a óbito e o outro melhorou após remoção cirúrgica do carcinoma de células escamosas e uso do homeopático Viscum album (Viscum Center ${ }^{\circledR}$ ). Entretanto, a utilização da AP no tratamento de efeitos adversos de drogas citotóxicas tem sido incrementada, pelo fato de que a ação da AP sobre o sistema imunológico ter sido evidenciada em diversos estudos, o que sugere a eficácia dessa técnica no tratamento da imunossupressão quimioterápica ${ }^{(33)}$.

O felino com carcinoma pulmonar veio a óbito. Dos dois felinos com diabetes um melhorou e o outro estava estável. O paciente com anemia hemolítica imunomediada melhorou. Nove pacientes apresentaram mais de uma afecção simultaneamente, seis dos quais afetando três sistemas orgânicos. $\mathrm{O}$ tratamento conjunto teve em conta a queixa principal e respectiva doença que colocava em risco a qualidade de vida do paciente.

O agulhamento manual foi a técnica mais usada $(94,7 \%)$, seguida pela EA $(30,6 \%)$ e a laserpuntura

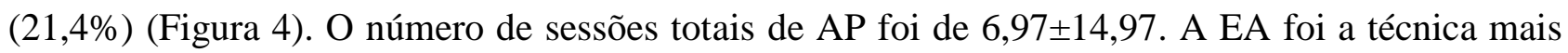
usada em felinos com doenças neurológicas (40,4\%) seguida da AP simples $(28,8 \%)$ e do LASER $(21,1 \%)$, de forma associada à AP/EA ou não. A agulha seca foi utilizada em cinco pacientes com problemas urinários, e num deles associou-se o LASER. 


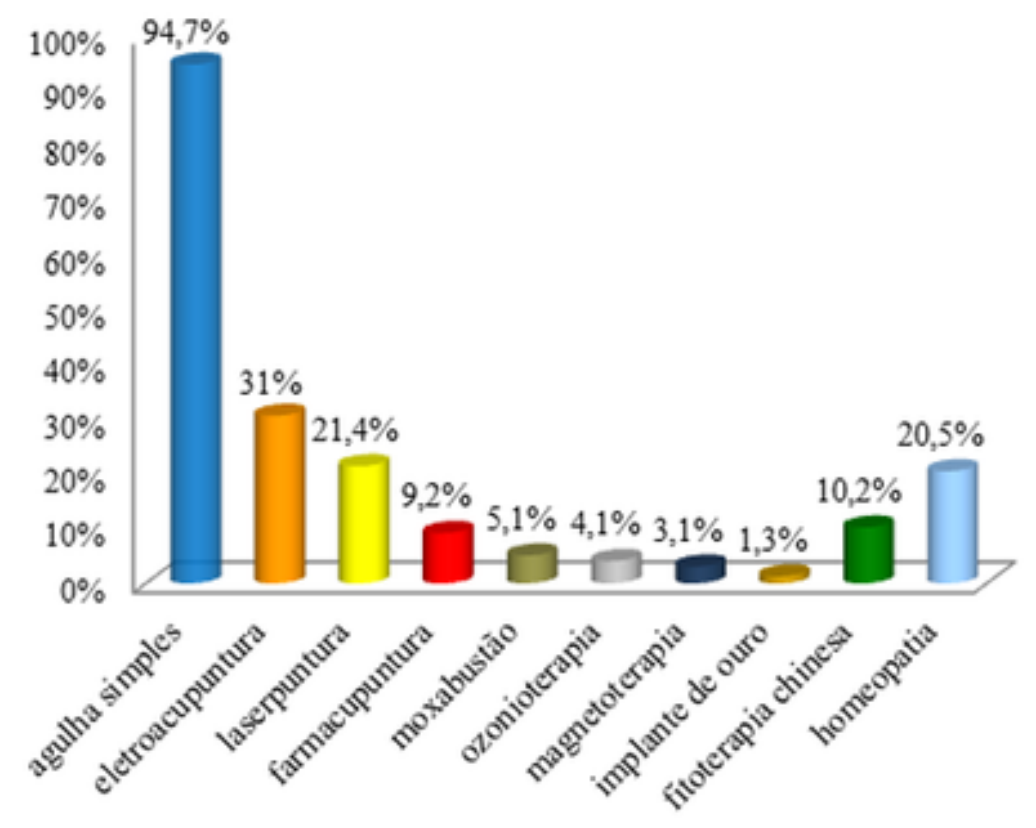

Figura 4 - Técnicas associadas ao tratamento com AP dos felinos atendidos no serviço de reabilitação e dor crônica entre 2000 e 2013. Os dados estão expressos em percentual dentro do universo de 98 animais.

A maioria dos felinos possui $Q i$ superficial e acessível e necessita apenas de agulhas curtas, pequenas e de estimulação leve que podem ser usadas nos pontos distais e pontos fontes-Yuan ${ }^{(34)}$. Nesta espécie podem-se utilizar agulhas de $127 \mathrm{~mm}$ de comprimento e $0,2 \mathrm{~mm}$ de diâmetro para os pontos Shu da Bexiga e 0,16-0,22 mm de diâmetro para os demais pontos ${ }^{(35)}$. Recomenda-se o uso de agulhas descartáveis a cada uma ou duas sessões, pois a sua reutilização torna a inserção desconfortável. A inserção de agulhas nos felinos deve ser rápida, a fim de evitar reações agressivas desencadeadas pela sensação (de-Qi) decorrente da sua inserção no paciente.

Em animais que não estão habituados à $\mathrm{AP}$, devem ser usados em primeiro lugar pontos localizados na região dorsal e, após a obtenção de relaxamento (5-10 min), podem ser agulhados pontos que geralmente causam mais incômodo, como os das extremidades, face e região ventral ${ }^{(36)}$. Em estudo com RM, verificou-se que a diferença de peso do corpo dos felinos teve diferença significativa na profundidade de segurança de alguns acupontos selecionados em felinos ${ }^{(37)}$.

A EA é a técnica mais empregada para indução de analgesia. O estímulo elétrico de acupontos é efetivo no alívio da dor central e periférica ${ }^{(38)}$. Na MTO, a EA tem sido utilizada para obtenção de hipoalgesia, devido à dificuldade de estimulação manual da agulha nos pacientes. Os cães adaptamse mais facilmente à técnica, enquanto os felinos apresentam reações diversas, com resultados favoráveis ou não ${ }^{(39)}$. Em felinos, a EA deve ser usada aumentando-se gradualmente a amplitude de corrente até um nível abaixo do limiar de conforto do animal ${ }^{(35)}$.

Trabalhos experimentais em gatos demonstram os efeitos da EA sobre as funções neurodegenerativas, rastreando os mediadores e regiões do $\mathrm{SN}$ envolvidos nos efeitos. Muitas destas pesquisas foram úteis, mas são muito discutíveis do ponto vista ético ${ }^{(40-46)}$.

Em gatas submetidas à ovariohisterectomia $(\mathrm{OH})$, a EA em E36 e vesícula biliar (VB) 34 diminuiu o consumo de anestésico e promoveu estabilidade cardiorrespiratória, sendo superior à AP falsa sem 
$\mathrm{EA}^{(47)}$ e ao uso da morfina ${ }^{(48)}$. A estimulação dos nervos medianos (simulando EA no PC6) melhorou a contratilidade cardíaca em gatos submetidos à isquemia do miocárdio ${ }^{(49)}$. Em um modelo felino, o efeito inibitório daquela estimulação na resposta pressora induzida pela bradicinina e a consequente melhora da disfunção isquêmica indicam que ela é dependente da ativação de receptores de opioides $^{(50)}$.

A injeção de fluidos nos acupontos prolonga a sua estimulação e é mais rápida e adequada para animais que não suportam o tempo necessário para a manutenção das agulhas ou a aplicação da EA. A farmacopuntura é a administração de subdoses de fármacos em pontos de AP, com potencialização do efeito do fármaco, minimização de efeitos colaterais e redução de custos ${ }^{(51)}$. Em cinco gatos atendidos utilizou-se a vitamina B12, em três usou-se lidocaína (1/10 da dose em casos de bexiga neurogênica) e em um insulina em E36 (diabetes). Pode-se aplicar vitamina B12 diluída em solução fisiológica (para diminuir a sensação de ardor), em volumes de 0,3 a $1 \mathrm{ml}$ por ponto. Lakshmipathi ${ }^{(52)}$ obteve sucesso com a AP para o tratamento de paraparesia, paralisia e paraplegia associada a injeções de vitamina B12, B1 e B6 em cães e gatos. A farmacopuntura com B12 tem especial recomendação em pacientes com DRC e doença inflamatória intestinal. A aplicação de B12 no acuponto VG20 é também usada em felinos com periúria que urinam em local inapropriado ${ }^{(53)}$.

A aquapuntura é a injeção de água destilada ou solução de $\mathrm{NaCl}$ a $0,9 \%$ nos acupuntos. No caso de animais com padrão de temperamento Fogo e com Calor Verdadeiro, segundo a MTO, sugere-se a aplicação de aquapuntura com 0.5 a $1 \mathrm{ml}$ de solução salina no ponto An-shen ${ }^{(34)}$. A eficácia analgésica de meloxican quando comparado com a farmacopuntura com meloxicam e aquapuntura de forma preemptiva em gatas submetidas à $\mathrm{OH}$, foi a mesma, sendo que para tal efeito utilizaram-se os acupontos Baço Pâncreas (BP) 6, VB34, Fígado (F) 3 e E36 ${ }^{(54)}$. A aquapuntura produziu analgesia residual de até 5 h após a $\mathrm{OH}$ em cadelas ${ }^{(55)}$.

Felinos que não toleram agulhas secas podem ser tratados com LASER ou moxabustão. Foram atendidos 21 pacientes com LASER. A laserpuntura é uma técnica não invasiva que consiste na utilização de LASER em acupontos e que apresenta efeito anti-inflamatório e analgésico em cães ${ }^{(56)}$ e gatos ${ }^{(57)}$. Consoante os casos, as agulhas são mantidas no animal por cinco a 25 minutos. A laserterapia de baixa potência é um recurso fisioterapêutico que favorece o retorno precoce das funções de membros lesionados. Esta técnica exerceu efeito benéfico sobre a articulação femorotibiopatelar de felinos no tratamento da ruptura do ligamento cruzado cranial ${ }^{(58)}$. Xie \& Preast $^{(35)}$ sugerem que o tratamento de felinos de constituição Água ou Madeira pode ser iniciado com o ponto Bai Hui (BH) e de felinos Terra ou Metal com o ponto VG20 ${ }^{(34)}$.

A moxabustão foi utilizada em cinco felinos e consiste no aquecimento de acupontos com a combustão da planta Artemisia vulgaris. A associação da AP com a moxabustão melhorou os sintomas da $\mathrm{OA}^{(59)}$. A moxa é muito eficaz em felinos com artropatias crônicas cujos sintomas pioram com o clima frio e úmido. Pode ser usada em todos os casos de Deficiência de Yang, conforme padrão da MTO. Esta técnica alternativa pode ser ensinada ao proprietário para aplicação em seu domicílio $^{(35)}$.

A ozoniopuntura (aplicação de ozônio nos acupontos em baixas concentrações) foi utilizada em dois felinos e a administração intra-retal em outros dois. A ozonioterapia é uma técnica promissora para tratar a dor, uma vez que a insuflação intra-retal de ozônio e a ozoniopuntura foram tão eficazes como o meloxicam para analgesia pós-operatória em cadelas submetidas à $\mathrm{OH}^{(60)}$. $\mathrm{O}$ aparelho de 
magnetoterapia foi usado em três felinos. O campo magnético produz relaxamento muscular e tem efeito analgésico na OA, por ação sobre terminações nervosas e liberação de endorfinas ${ }^{(61)}$. No pósoperatório tem efeito anti-inflamatório, por redução do edema e dos mediadores químicos próinflamatórios ${ }^{(62)}$.

O implante de fragmentos de ouro foi aplicado em dois felinos com paraparesia devido a fratura medular. Um deles melhorou gradualmente a deambulação; o outro não retornou. O implante de ouro tem sido usado para uma estimulação prolongada dos acupontos, particularmente em casos de doença articular degenerativa e DCF. O seu mecanismo de ação é, em parte, anti-inflamatório ${ }^{(63)}$.

Em nove felinos foram associados fitoterápicos ao tratamento. Em muitos casos, o tratamento eficaz em MTO depende do uso da fitoterapia chinesa, a qual funciona como um suplemento alimentar ou nutracêutico. Para tratar de um animal com AP e fitoterapia chinesa, é necessário descrever seus sintomas para enquadrá-los em padrões e assim diagnosticar suas desarmonias segundo a MTO. Os compostos são aceitos pela maioria dos felinos, na forma líquida (tinturas), em pó de amido (ou lactose) ou em cápsulas ${ }^{(28)}$. Para diversas afecções, 26 felinos foram tratados com homeopatia, dois dos quais exclusivamente, outro em associação com fitoterapia. O uso de remédios homeopáticos, escolhidos de acordo a totalidade de sinais do caso após uma completa anamnese homeopática, é uma ferramenta valiosa no tratamento de hipertireoidismo felino e pode ser associada à AP e à fitoterapia $^{(64)}$.

\section{Conclusões}

A AP foi eficiente para tratar a maioria dos gatos com diferentes condições clínicas, mostrando que esta espécie provavelmente responde tão bem como os cães após o tratamento de AP. Em caso de animais com doenças com mau prognóstico com AP, os proprietários devem ser aconselhados que este tipo de tratamento pode ser usado como uma ferramenta para a manutenção da qualidade de vida.

\section{Referências}

1. Lopes AA. Medicina Baseada em Evidências: a arte de aplicar o conhecimento científico na prática. Revista da Associação Médica Brasileira. 2000;46(3):285-288.

2. Taffarel MO, Freitas PMC. Acupuntura e analgesia: aplicações clínicas e principais acupontos. Ciência Rural. 2009;39(9):2665-2672.

3. Luna SPL, Joaquim JGF. Effect of electroacupuncture (EA) on gastrointestinal peristaltism in dogs. Brazilian Journal of Veterinary Research and Animal Science. 2004;41(Supl):150.

4. Joaquim JG, Morais R, Miranda JRA, Luna SPL. Effect of acupuncture/eletroacupuncture on gastric pressure and contraction of dogs measured by alternate current susceptometry and manometry. ARS Veterinaria. 2008;24(1):9-14.

5. Janssens L, Altman S, Rogers PA. Respiratory and cardiac arrest under general anaesthesia: treatment by acupuncture of the nasal philtrum. Veterinary Record. 1979;105(22):273-276. 
6. Bazolli RS. Efeito da acupuntura sobre arritmias ventriculares induzidas com cloreto de bário em cães [Dissertação de Mestrado]. Jaboticabal: Faculdade de Ciências Agrárias e Veterinárias da Universidade Estadual Paulista; 2003.

7. Luna SPL, Joaquim JGF, Gama ED. Immunomediated thrombocytopenia treated with acupuncture and Chinese herbs. In: Proceedings of the $30^{\text {th }}$ Annual International Congress on Veterinary Acupuncture, 2004, Sep 8-11; Oostende, Belgium. Red Hook: Curran \& Associates. p. 311-319.

8. Joaquim JGF, Luna SPL, Torelli S, Angeli AL. Acupuntura como tratamento de doenças neurológicas em cães. Revista Acadêmica, Ciência Animal. 2008;6(3):327-334. DOI: http://dx.doi.org/10.7213/cienciaanimal.v6i3.10574. Acesso em janeiro de 2018.

9. Ueda MY, Luna SPL, Joaquim JGF, Scognamillo Szabó MVR. Estudo retrospectivo de 1137 animais submetidos à acupuntura na FMVZ-Unesp-Botucatu-SP. ARS Veterinaria. 2010;26(1):6-10.

10. Associação Brasileira da Indústria de Produtos para Animais de Estimação - ABINPET. 2011. [acesso 2013 Dez 20]. Disponível em: http://abinpet.org.br/imprensa/noticias/abinpet-divulga-mediade-gasto-com-pets/

11. Yang JW, Jeong SM, Seo KM, Nam TC. Effects of corticosteroid and electroacupuncture on experimental spinal cord injury in dogs. Journal of Veterinary Science. 2003;4(1):97-101.

12. Hayashi AM, Matera JM, Pinto AC. Evaluation of electroacupuncture treatment for thoracolumbar intervertebral disk disease in dogs. Journal of the American Veterinary Medical Association. 2007;231(6):913-918.

13. Joaquim JG, Luna SP, Brondani JT, Torelli SR, Rahal SC, de Paula Freitas F. Comparison of decompressive surgery, electroacupuncture, and decompressive surgery followed by electroacupuncture for the treatment of dogs with intervertebral disk disease with long-standing severe neurologic deficits. Journal of the American Veterinary Medical Association. 2010;236(11):1225-1229.

14. Joaquim JGF. Uso da acupuntura nas síndromes articulares: primeira ou última opção? Acta Scientiae Veterinariae. 2007;35(Supl 2):S277-S278.

15. Altman S. Application of Acupuncture to Neurologic Diseases. In: Proceedings of XX Congress of the World Small Animal Veterinary Association. 1995; Yokohama, Japan.

16. Choi KW, Hill SA. Acupuncture treatment for feline multifocal intervertebral disc disease Case report. Journal of Feline Medicine and Surgery. 2009;11(8):706-710.

17. Schoen AM. Acupuncture for musculoskeletal disorders. Problems in Veterinary Medicine. 1992;4(1):88-97.

18. Lascelles BDX, Robertson SA, Gaynor JS. Can Chronic Pain in Cats be Managed? Yes! In: Proceedings of VIN's Managing Pain Symposium. Managing Pain in Cats, Dogs, Small Mammals and Birds - recognition, relief and economics Symposim, 2003. [acesso 2013 Mar 31]. Disponível em: www.vin.com

19. Angeli AL, Joaquim JGF, Gama ED, Luna SPL. Outcome of 119 dogs and cats treated at the acupuncture unit of the Faculty of Veterinary Medicine and Animal Science of the University of São Paulo State, Botucatu city, Brazil. Brazilian Journal of Veterinary Research and Animal Science. 2005;42(1):68-74. 
20. Steiss JE. Canine rehabilitation. In: Steiss JE, Braund KG, ed. Clinical neurology in small animals: localization, diagnosis and treatment. Ithaca, New York: International Veterinary Information Service; 2002. p. 145-157.

21. Pelizzari C, Mazzanti A, Raiser AG, Lopes STA, Graça DL, Salbego FZ, et al. Medium frequency neuromuscular electrical stimulation (russian) in dogs with induced muscle atrophy. Ciência Rural. 2008;38(3):736-742.

22. Fernández VL, Bernardini M. Enfermidades da Medula com Afecção Neurológica Secundária. In: Fernández VL, Bernardini M. Neurologia de cães e gatos. São Paulo: Med Vet; 2010. p.319-366.

23. Vicente AG. Neurología Veterinaria en el Perro y el Gato. $1^{\mathrm{a}}$ ed. Barcelona: Pulso ediciones; 1998.

24. Bussel B, Roby-Brami A, Néris OR, Yakovleff A. Evidence for a spinal stepping generator in man. Paraplegia. 1996;34(2):91-92.

25. Fowler ME, Miller RE. Fowler's Zoo and Wild Animal Medicine, Current Therapy. $4^{\text {th }}$ ed. Philadelphia: W.B. Saunders; 1999. p. 657-662.

26. Wright BD. Clinical pain management techniques for cats. Clinical Techniques in Small Animal Practice. 2002;17(4):151-157.

27. Taylor PM, Robertson SA. Pain management in cats--past, present and future. Part 1 . The cat is unique. Journal of Feline Medicine and Surgery. 2004;6(5):313-320.

28. Beebe S, Salewski M, Monda L, Scott J. Clinical Handbook of Chinese Veterinary Herbal Medicine. Placitas: Herbal Medicine Press. $1^{\text {st }}$ ed. 2006. 208 p.

29. Giovaninni LH, Piai VS. O uso da acupuntura no auxílio à terapia da doença idiopática do trato urinário inferior dos felinos. Ciência Rural. 2010;40(3):712-717.

30. Yang J, Wang C. Electroacupuncture at "Zusanli"(ST 36) can inhibit frequencies of transient lower esophageal sphincter relaxation induced by gastric distention in cats. Zhen Ci Yan Jiu: Acupuncture Research. 2011;36(6):423-427.

31. Wang C, Zhou DF, Shuai XW, Liu JX, Xie PY. Effects and mechanisms of electroacupuncture at PC6 on frequency of transient lower esophageal sphincter relaxation in cats. World Journal of Gastroenterology. 2007;13(36):4873-4880.

32. Shuai X, Xie P, Liu J, Xiang Y, Li J, Lan Y. Different effects of electroacupuncture on esophageal motility and serum hormones in cats with esophagitis. Diseases of the Esophagus. 2008;21(2):170175 .

33. Cápua MLB. Acupuntura no tratamento da imunossupressão quimioterápica [Monografia]. apresentada ao Programa do Curso de Especialização de Acupuntura Veterinária, Universidade Estadual Júlio de Mesquita Filho - UNESP. 2006. 25 p.

34. Xie H. Acupuncture in Cats: Is There a Difference From Dogs? Proceedings of the $34^{\text {th }}$ World Small Animal Veterinary Congress WSAVA 2009. São Paulo; 2009.

35. Xie H, Preast V. Acupuntura Veterinária Xie. São Paulo: Med Vet; 2011.

36. Marsden S. Acupuncture for Feline Disorders. Basic course in veterinary acupuncture. $22^{\text {nd }}$ ed. 
Course notes IVAS 2012 - 2013. Atlanta; 2012 p. 695-715.

37. Lin J. Determination of the Safe Depth of Back Acupoints in Cats: Investigation Using 117 Magnetic Resonance Imaging, 2004. In: Proceedings of the $30^{\text {th }}$ Annual International Congress on Veterinary Acupuncture, 2004, Sep 8-11; Oostende, Belgium. Red Hook: Curran \& Associates. p. 116-117.

38. Liu HX, Tian JB, Luo F, Jiang YH, Deng ZG, Xiong L, et al. Repeated $100 \mathrm{~Hz}$ TENS for the Treatment of Chronic Inflammatory Hyperalgesia and Suppression of Spinal Release of Substance P in Monoarthritic Rats. Journal of Evidence-Based Complementary \& Alternative Medicine. 2007;4(1):65-75.

39. Still J. Acupuncture analgesia for laparotomy in dogs and cats: an experimental study. American Journal of Acupuncture. 1987;15:155-165.

40. Goto T, Hoshino Y. Electrophysiological, histological, and behavioral studies in a cat with acute compression of the spinal cord. Journal of Orthopaedic Science. 2001;6(1):59-67.

41. Li P, Tjen-A-Looi S, Longhurst JC. Rostral ventrolateral medullary opioid receptor subtypes in the inhibitory effect of electroacupuncture on reflex autonomic response in cats. Autonomic Neuroscience: Basic and Clinical. 2001;89(1-2):38-47.

42. Liu F, Sun WW, Wang Y, Hu LQ, Dai P, Tian CF, et al. Effects of electro-acupuncture on NT-4 expression in spinal dorsal root ganglion and associated segments of the spinal dorsal horn in cats subjected to adjacent dorsal root ganglionectomy. Neuroscience Letters. 2009;450(2):158-162.

43. Sun WW, Zhao W, Wang TH. Effects of electro-acupuncture on PDGF expression in spared dorsal root ganglion and associated dorsal horn subjected to partial dorsal root ganglionectomy in cats. Neurochemical Research. 2008;33(3):437-443.

44. Tai C, Booth AM, de Groat WC, Roppolo JR. Bladder and urethral sphincter responses evoked by microstimulation of $\mathrm{S} 2$ sacral spinal cord in spinal cord intact and chronic spinal cord injured cats. Experimental Neurology. 2004;190(1):171-183.

45. Wang TT, Yuan WL, Ke Q, Song XB, Zhou X, Kang Y, et al. Effects of electro-acupuncture on the expression of $\mathrm{c}$-jun and $\mathrm{c}$-fos in spared dorsal root ganglion and associated spinal laminae following removal of adjacent dorsal root ganglia in cats. Neuroscience. 2006;140(4):1169-1176.

46. Wang TH, Wang XY, Li XL, Chen HM, Wu LF. Effect of electroacupuncture on neurotrophin expression in cat spinal cord after partial dorsal rhizotomy. Neurochemical Research. 2007;32(8):1415-1422.

47. Melo MS. Efeito da eletroacupuntura em gatas (felis catus domesticus) anestesiadas por isofluorano e submetidas à ovariosalpingohisterectomia [Dissertação de mestrado]. Uberlândia: Faculdade de Medicina Veterinária. Universidade Federal de Uberlândia; 2005. 54 p.

48. Freitas PMC, Pignaton W, Simões JR, Zacché E, Luz MR, Salgado AEP, et al. Eletroacupuntura e morfina sobre parâmetros cardiorrespiratórios em gatas submetidas à ovariosalpingohisterectomia eletiva. Revista Brasileira de Saúde e Produção Animal. 2011;12(4):961-969.

49. Li P, Pitsillides KF, Rendig SV, Pan HL, Longhurst JC. Reversal of reflex-induced myocardial ischemia by median nerve stimulation: a feline model of electroacupuncture. Circulation. 
1998;97(12):1186-1194.

50. Chao DM, Shen LL, Tjen-A-Looi S, Pitsillides KF, Li P, Longhurst JC. Naloxone reverses inhibitory effect of electroacupuncture on sympathetic cardiovascular reflex responses. American Journal of Physiology. 1999;276(6 Pt 2):H:2127-2134.

51. Luna SP, Angeli AL, Ferreira CL, Lettry V, Scognamillo-Szabó M. Comparison of pharmacopuncture, aquapuncture and acepromazine for sedation of horses. Journal of EvidenceBased Complementary \& Alternative Medicine. 2008;5(3):267-272.

52. Lakshmipathi GV. Acupuncture Therapy for Neuromuscular Disorders in Pet Animals (Dogs \& Cats). In: Proceedings of the $26^{\text {th }}$ Annual International Congress on Veterinary Acupuncture; 2000; Vienna, Austria.

53. Robinson NG. Veterinary acupuncture: an ancient tradition for modern times. Alternative and Complementary Therapies. 2007;13(5):259-265.

54. Sousa NR, Luna SPL, Cápua MLB, Lima AFM, Oliveira FA, Viveiros BM, et al. Analgesia of preemptive pharmacopuncture with meloxicam or aquapuncture in cats undergoing ovariohysterectomy. Ciência Rural. 2012;42(7):1231-1236.

55. Luna SPL, Kelawala NH, Lima AFM, Saarto EE, Restitutti FC, Silva NEOF. Effect of aquapuncture on the postoperative analgesia after ovariohysterectomy in dogs. Semina: Ciências Agrárias. 2015;36(3 Supl. 1):1979-1990.

56. Taffarel MO, Cardoso GS, Luna SPL, Derussi AAP, Volpato R, Guaitolini CRF, et al. Preemptive analgesia with laserpuncture in dogs undergoing ovariohysterectomy. Vet Anaesth Analg. 2013;40(5):4. Acessed in http://onlinelibrary.wiley.com/doi/10.1111/vaa.12053/full.

57. Marques VI, Cassu RN, Nascimento FF, Tavares RC, Crociolli GC, Guilhen RC, et al. Laser Acupuncture for Postoperative Pain Management in Cats. Journal of Evidence-Based Complementary \& Alternative Medicine. 2015:653270.

58. Mantovani PF. Laserterapia de baixa potência no tratamento da ruptura do ligamento cruzado cranial em felinos (felis catus). Anais do IX Congresso Brasileiro de Cirurgia e Anestesiologia Veterinária. Jornal Brasileiro de Ciência Animal. 2010;3(6):190. Disponível em http://www.jbca.com.br/v3n6/reportagem/anais_cbcav_final.pdf, acesso em janeiro 2018.

59. Zhang QR, Fu WB. Osteoarthritis of knee joint treated with acupuncture and moxibustion. Zhongguo Zhen Jiu. 2010;30(5):375-378.

60. Teixeira LR, Luna SP, Taffarel MO, Lima AF, Sousa NR, Joaquim JG, et al. Comparison of intrarectal ozone, ozone administered in acupoints and meloxicam for postoperative analgesia in bitches undergoing ovariohysterectomy. Veterinary Journal. 2013;197(3):794-799.

61. Robertson JA, Juen N, Théberge J, Weller J, Drost DJ, Prato FS, et al. Evidence for a dosedependent effect of pulsed magnetic fields on pain processing. Neuroscience Letters. 2010;482(2):160-162.

62. Rohde C, Chiang A, Adipoju O, Casper D, Pilla AA. Effects of pulsed electromagnetic fields on interleukin-1 beta and postoperative pain: a double-blind, placebo-controlled, pilot study in breast reduction patients. Plastic and Reconstructive Surgery. 2010;125(6):1620-1629. 
63. Jaeger GT, Larsen S, Søli N, Moe L. Double-blind, placebo-controlled trial of the pain-relieving effects of the implantation of gold beads into dogs with hip dysplasia. Veterinary Record. 2006;158(21):722-726.

64. Chapman SF. Homeopathic and integrative treatment for feline hyperthyroidism--four cases (2006-2010). Homeopathy. 2011;100(4):270-274. 